\title{
Color Linear Model
}

Chang-Yeong Kim*, Yang-Seok Seo*, In-So Kweon ${ }^{\circ}$

* Signal Processing Lab. Samsung Advanced Institute of Technology, P.O. Box 111, Suwon, Korea 440-600 Phone) 0331-280-9217 fax) 0331-280-9207 Cykim@saitgw.sait.samsung.co.kr - Dept. of Electrical Engineering, Korea Advanced Institute of Science and Technology, 207-43, CheongRyangRiDong, DongDaeMoonGu. Seoul,Korea,130-012

Phone) 02-985-3415 fax)02-968-1638 Kweon(ädesign.kaist.ac.kr

\begin{abstract}
In this paper, procedures for creating an effective linear model to represent surface spectra are presented. The model is derived by considering spectral data and the human visual characteristic that depends on wave lengths. Two human visual weighting functions (HVWF) are derived from human visual characteristic. The basis functions of the linear model for the surface reflectance are selected by minimizing least square error in approximating the spectral data weighted by the HVWF. The linear model is shown to perform better than conventional linear models for color constancy, the surface identification related to object recognition, and the characterization of a scanner and a camera.
\end{abstract}

\section{Introduction}

Recent computational models of color vision [1-7] demonstrate that it is possible to achieve color constancy over some limited range of illuminant and surface. The algorithm provided by Cohen [1] initially was attempted to find an efficient, low dimensional linear representation of surface reflectance (SR) and spectral power distribution (SPD) of illuminants. Efficient spectral representation is useful in many applications such as color reproduction system $[4,5]$, rendering in computer graphics, color constancy, and surface identification related to object recognition.

Linear model has been developed in two main directions. First, relationship between the error of reconstructed reflectance and the dimension of basis functions has been observed in the linear model $[2,3,7]$. Second is to analyze not only the error of reconstructed reflectance but also color difference on color space for real applications $[6,10,11]$ A linear model designed to minimize the error between original $\mathrm{SR}$ and reproduced one is inappropriate in efficient color representation because it is not designed to minimize color differences. Moreover, it is important to remember that basis functions should be derived independent of illuminants because color constancy is get "an invariant" under the varying illuminants. Therefore, we try to consider and minimize the human visual characteristic (HVC) independent of illuminant to derive the basis function. In [10], the effectiveness of basis functions considering HVC was proved. In this paper, the CIE $1931 \mathrm{XYZ}$ standard observer and CIE1976 CIEL*a*b* as HVC are investigated.

\section{Linear Model}

Linear models [1-8] are used to approximate SR with a small number of descriptors in wavelength domain. The basis functions $R_{j}(\lambda)$ for $\mathrm{SR}$ are chosen to minimize the mean square error of reflectance $R(\lambda)$ :

$$
{\underset{R}{R_{j}} \underset{S \in \text { sample }}{\sum} \int} \int\left[R_{s}(\lambda) \cdot \sum_{j=1}^{d} \sigma_{j}(\lambda) R_{j}(\lambda)\right]^{2} d \lambda .
$$


where sample and $d$ represent the number of samples and basis functions respectively and $\sigma_{\mathrm{j}}$ is the weight of each basis function. If the $d$-dimensional basis functions are chosen to approximate the $\operatorname{SR} R(\lambda)$, the reflectance function is represented as:

$$
R(\lambda) \approx \sum_{j=1}^{d} \sigma_{j} R_{j}(\lambda) \equiv R^{\prime}(\lambda)
$$

The basis functions $R_{\mathrm{j}}$ that minimize the quantity in equation (1) are derived by as singular value decomposition (SVD) of the matrix [9] whose columns are composed of the SR of samples [1,5,6]. Let us consider SR as 31 by 1 matrix with $10 \mathrm{~nm}$ sampling wavelengths from $400 \mathrm{~nm}$ to $700 \mathrm{~nm}$. If mapping of the original reflectance matrix, $R$, onto the reconstructed spectral reflectance matrix $\boldsymbol{R}^{\prime}$ is expressed by the projection matrix:

$$
R^{\prime}=R_{j} W=R_{j}\left(R_{j}^{+} R\right)=\left(R_{j} R_{j}^{+}\right) R=P R
$$

where $W$ is 3 by 1 weight matrix,$R_{j}^{+}$is the psudoinverse of the basis vectors and $P$ is the projection matrix with entries of 31 by 31 . The mean square error, $E_{\text {ref }}$, between the original and an approximated spectra become:

$$
E_{r e f}=\frac{1}{31} \sum_{j=1}^{3 i}\left(\left|R\left(\lambda_{j}\right)-R^{\prime}\left(\lambda_{j}\right)\right|^{2}\right)=\|R-P R\|^{2}
$$

where 31 is the number of sampling points in spectrum and \|\| represents the norm of vector. The linear model for illuminant has the same representation as for reflectance except that basis vectors derived from a set of illuminants used. The linear approximation $E(\lambda)$ for illuminant corresponding to equation (2) is $E(\lambda) \approx \sum_{j=1}^{d} E_{j}(\lambda) \varepsilon_{j}$, where $E_{j}(\lambda)$ are basis functions for illuminant set.

\section{Color Linear Model using Human Visual Perception}

It is important that the magnitude of spectra errors is not always coincident with the amount of color difference perceived by human eye, such as CIEXYZ or CIELAB:

$$
\text { if }\left(\Delta E_{r e f}^{a}<\Delta E_{r e f}^{b}\right) \text {, then }\left(\Delta E_{a b}^{* a}>\Delta E_{a b}^{* b}\right) \text { or }\left(\Delta E_{a b}^{* a}<\Delta E_{a b}^{* b}\right) \text {. }
$$

Basically, this inequivalence is due to minimization strategy, equation (1), in which the HVC is not considered. Human visual responses are relatively insensitive at the ends of visible spectrum, while they show different sensitivity characteristics in around the center of visible spectrum. The color constancy, perceived by human visual system, for an object discounts illumination effect not for reflectance but for trichromatic visual response. Wandell et al. derived the basis vectors of color data set by $n$-mode analysis to minimize the error in the predicted scanner response [6]. Trusell has discussed the related topic that uses principal component analysis (PCA) in color reproduction [5]. The target of this section is to derive the basis functions considering the human visual perception for the surface reflectance set.

\subsection{The Proposed Minimization Equation}

To derive effective basis functions, the error is minimized for reflectance data based on human characteristic rather than SR itself : 
$\min \sum_{\text {sample }} \llbracket\left[H(\lambda)-H^{\prime}(\lambda)\right]^{2} d \lambda=\min \sum_{\text {sample }} \int\left[H(\lambda)-H_{j}(\lambda) \cdot \sigma_{h j}(\lambda)\right]^{2} d \lambda$

where $H(\lambda)$ represent the product of reflectance $R(\lambda)$ and $H V C$, and $H^{\prime}(\lambda)$ is an approximated reflectance in human visual domain and $\sigma_{h j}$ is weight coefficient and $H_{j}(\lambda)$ are basis functions. The basis functions $H_{j}$ for the SR are chosen to minimize the mean square error of $\mathrm{SR}$ data $H(\lambda)$. If reflectance replaces the continuous function of wave length with sampled functions expressed as vectors, then the human visual error as in equation (4):

$$
E_{H V}=\left\|\boldsymbol{H}-\boldsymbol{P}_{H} \boldsymbol{H}\right\|
$$

where $\boldsymbol{P}_{\mathrm{H}}$ is a projection matrix and \|\| represents the norm of vector.

\subsection{Human Visual Weighting Functions (HVWF)}

Two examples of HVWF described here are found from the three spectral components of color vector such as CIEXYZ or CIELAB. All spectral data are composed of 31 sample points ranging from 400 to $700 \mathrm{~nm}$ in 10-nm steps in our model. Human visual sensitivities are $C_{i}, i=1-3$, whose entries compose of three column matrix, $C=\left[C_{l}, C_{2}, C_{3}\right]$. Three spectral human visual responses $X_{i j}$ of the surface reflectance $R$ under the illuminant $E$ at each sample wave length can be computed by matrix product $X=C^{\top} E R$, where $R$ and $E$ are 31 by 31 diagonal matrices. Hence, each column matrix of $X$ is expressed as:

$$
X_{j}=R\left(\lambda_{j}\right) E\left(\lambda_{j}\right)\left[C_{t}\left(\lambda_{j}\right), C_{2}\left(\lambda_{j}\right), C_{3}\left(\lambda_{j}\right)\right]^{\mathrm{T}}
$$

where $j=1-31$. Suppose $T(\lambda)$ as CIE 1931 Standard Observer [8], then human visual response at sample wave lengths becomes in terms of CIE $X Y Z$ values as:

$$
X_{j}=R\left(\lambda_{j}\right) E\left(\lambda_{j}\right) K T\left(\lambda_{j}\right),
$$

where $K=100 / \sum_{j=1}^{31}\left[E\left(\lambda_{j}\right) \cdot \bar{y}\left(\lambda_{j}\right) \cdot \Delta \lambda\right]$, and $T\left(\lambda_{j}\right)$ is a vector form of trichromatic observer, $\left[\bar{x}\left(\lambda_{j}\right), \bar{y}\left(\lambda_{j}\right), \bar{z}\left(\lambda_{j}\right)\right]^{\mathrm{T}}$. Suppose $\boldsymbol{X}_{j}^{\prime}$ the recovered human visual response of the reflectance $R\left(\lambda_{j}\right)$, then the norm of the difference of human response vector:

$$
\left\|X_{j}-X_{j}^{\prime}\right\|=E\left(\lambda_{j}\right) \mathrm{K}\left\{R\left(\lambda_{j}\right)-\mathrm{R}^{\prime}\left(\lambda_{j}\right)\right\}\left[\mathrm{T}_{i}\left(\lambda_{j}\right)^{2}+\mathrm{T}_{2}\left(\lambda_{j}\right)^{2}+\mathrm{T}_{3}\left(\lambda_{j}\right)^{2}\right]^{1 / 2}
$$

If we substitute the norm $\left\|X_{j}-X_{j}\right\|$ instead of $\left(R\left(\lambda_{j}\right)-R^{\prime}\left(\lambda_{j}\right)\right)$ in equations (1) and (7), then the basis functions can be chosen to minimize the following quantity:

$$
\sum_{\text {sample }} \sum_{j}\left[E\left(\lambda_{j}\right) \cdot K \cdot\left\|T\left(\lambda_{j}\right)\right\| \cdot\left\{R\left(\lambda_{j}\right)-R^{\prime}\left(\lambda_{j}\right)\right\}\right]^{2} \text {. }
$$

Using equation (11), we are ready to define the HVWF at sample wave lengths as:

$$
H_{v}\left(\lambda_{j}\right)=\mathrm{E}\left(\lambda_{j}\right) \mathrm{K}\left[\mathrm{C}_{1}\left(\lambda_{j}\right)^{2}+\mathrm{C}_{2}\left(\lambda_{j}\right)^{2}+\mathrm{C}_{3}\left(\lambda_{j}\right)^{2}\right]^{1 / 2}
$$

Equation (12) displays the HVWF of a general form considering the illuminant and HVC. Equation (11) can be rewritten by:

$$
\sum_{\text {sample } j} \sum_{j}\left[H_{v}\left(\lambda_{j}\right) R\left(\lambda_{j}\right)-H^{\prime}\left(\lambda_{j}\right)\right]^{2}
$$

Besides the above HVWF, it is possible to adopt a human visual sensitivity that is 
a linear space of the reflectance such as NTSC RGB or ATD space [8]. Suppose an illuminant with uniformed unit energy spectrum, then the HVWF that is independent of illuminant is expressed by:

$$
H_{v}\left(\lambda_{j}\right)=\left[C_{1}\left(\lambda_{j}\right)^{2}+C_{2}\left(\lambda_{j}\right)^{2}+C_{3}\left(\lambda_{j}\right)^{2}\right]^{1 / 2}, j=1, \ldots 31 .
$$

The above $H_{V}\left(\lambda_{j}\right)$ is depicted in Figure 1 and is refereed to color matching function (CMF) in the following sections. CMF represents characteristics that the middle range of visible spectrum has a high sensitivity like human being's but both ends of visible spectrum have relatively low sensitivity. Besides the linear spaces, non-linear space to reflectance like $\mathrm{LAB}$ (CIE $1976 \mathrm{~L}^{*} \mathrm{a}^{*} \mathrm{~b}^{*}$ ) space [8] can be considered as the HVC. The XYZ values $X_{j}$ of equation (9) can be transformed into LAB domain:

$$
\begin{aligned}
& \mathrm{L}\left(\lambda_{j}\right)=116\left\{\mathrm{R}\left(\lambda_{j}\right) \mathrm{E}\left(\lambda_{j}\right) \mathrm{K} \mathrm{T}_{2}\left(\lambda_{j}\right) / \mathrm{Yn}\right\}^{1 / 3}-16 \\
& \mathrm{~A}\left(\lambda_{j}\right)=500\left[\left\{\mathrm{R}\left(\lambda_{j}\right) \mathrm{E}\left(\lambda_{j}\right) \mathrm{K} \mathrm{T}_{1}\left(\lambda_{j}\right) / \mathrm{Xn}\right\}^{1 / 3}-\left\{\mathrm{R}\left(\lambda_{j}\right) \mathrm{E}\left(\lambda_{j}\right) \mathrm{K} \mathrm{T} \mathrm{T}_{2}\left(\lambda_{j}\right) / \mathrm{Yn}\right\}^{1 / 3}\right] \\
& \mathrm{B}\left(\lambda_{j}\right)=200\left[\left\{\mathrm{R}\left(\lambda_{j}\right) \mathrm{E}\left(\lambda_{j}\right) \mathrm{K} \mathrm{T}_{2}\left(\lambda_{j}\right) / \mathrm{Yn}\right\}^{1 / 3}-\left\{\mathrm{R}\left(\lambda_{j}\right) \mathrm{E}\left(\lambda_{j}\right) \mathrm{K} \mathrm{T} \mathrm{T}_{3}\left(\lambda_{j}\right) / \mathrm{Zn}\right\}^{1 / 3}\right]
\end{aligned}
$$

where $\mathrm{Xn}, \mathrm{Yn}$, and $\mathrm{Zn}$ represent $\mathrm{CIE} X, Y$, and $\mathrm{Z}$ values of a given illuminant. Let us $X_{j}$ a column matrix $\left[\mathrm{L}\left(\lambda_{j}\right), \mathrm{A}\left(\lambda_{j}\right), \mathrm{B}\left(\lambda_{j}\right)\right]^{\mathrm{T}}$. Suppose an uniformed illuminant, $E\left(\lambda_{j}\right)=[1]$, and human visual response $l\left(\lambda_{j}\right), a\left(\lambda_{j}\right)$, and $b\left(\lambda_{j}\right)$ for a surface with unit reflectance, then the norm of human visual difference vector between original and the estimated one become:

$$
\left\|X_{j}-X_{j}\right\|=\|T(\lambda)\|\left\{\mathrm{R}\left(\lambda_{j}\right)^{1 / 3}-\mathrm{R}^{\prime}\left(\lambda_{j}\right)^{1 / 3}\right\}
$$

where $\|T(\lambda)\|=\left\{l\left(\lambda_{j}\right)^{2}+a\left(\lambda_{i}\right)^{2}+b\left(\lambda_{i}\right)^{2}\right\}^{1 / 2}$. The quantity corresponding to equation (11) is then represented by:

$$
\sum_{\text {sample }} \sum_{j}\left\{\|T(\lambda)\|\left\{R\left(\lambda_{j}\right)^{1 / 3}-R^{\prime}\left(\lambda_{j}\right)^{1 / 3}\right\}\right\}^{2} .
$$

There are two obstacles to apply HVC with a non-linear color space in a linear model:

1) It is impossible to separate $\operatorname{SR} R\left(\lambda_{j}\right)$ from the minimization equation (19), hence human visual weighting function cannot be derived;

2) It is hard to understand $L A B$ values at sample wave lengths because $L A B$ values are defined from the sum of $X Y Z$ values along to wave length. Moreover, $L A B$ space is non-linear to reflectance space. Hence, there is difficult to apply LAB values as the HVC in the linear model directly, even though the color space describes the HVC quite well. Hence, assuming a recovered human visual response $H^{\prime}\left(\lambda_{j}\right)$, then HVWF can be derived by minimizing a different quantity from equation(19):

$$
\sum_{\text {sample } j} \sum_{j}\left[\|T(\lambda)\|^{1 / p} \cdot R\left(\lambda_{j}\right)-H^{\prime}\left(\lambda_{j}\right)\right]^{2}
$$

where $\|\boldsymbol{T}(\lambda)\|^{1 / \mathrm{p}}$ is p-norms defined by $\left(\left|T_{1}\right|^{p}+\left|T_{2}\right|^{p}+\left|T_{3}\right|^{p}\right)^{1 / p}, p \geq 1$.

Here, we try to define a quantity similar to equation (19) that uses the p-norms of LAB values and extracts $p$ by minimizing the total error for variables. HVWF at sample wave lengths in the above equation is defined by:

$\operatorname{HVWF}\left(\lambda_{j}\right)=\left[\left\{L\left(\lambda_{j}\right)\right\}^{\mathrm{P}}+\left\{\mathrm{A}\left(\lambda_{j}\right)\right\}^{\mathrm{P}}+\left\{\mathrm{B}\left(\lambda_{j}\right)\right\}^{\mathrm{P}}\right]^{\mathrm{L} / \mathrm{P}}$.

To get more information about $\angle A B P$ the original spectra are reconstructed and the errors are estimated for the dimension of basis vector from three to seven. The errors of the reconstructed spectra decrease as the number of basis increases from three to seven while the maximum errors in $\mathrm{XYZ}$ space increase. This is due to minimize not 
$X Y Z$ error but the error of weighted spectra of $\mathrm{LAB}_{\mathrm{P}=2}$ for deriving the linear model. The basis vector of parameter $p=2$ gives the best reconstruction for spectra, $X Y Z$, and LAB. Figure 1 shows three kinds of HVWFs that are uniform, CMF, and LAB the case of $p=2$, where the $L A B$ has three maximum points that is different from $C M F$.

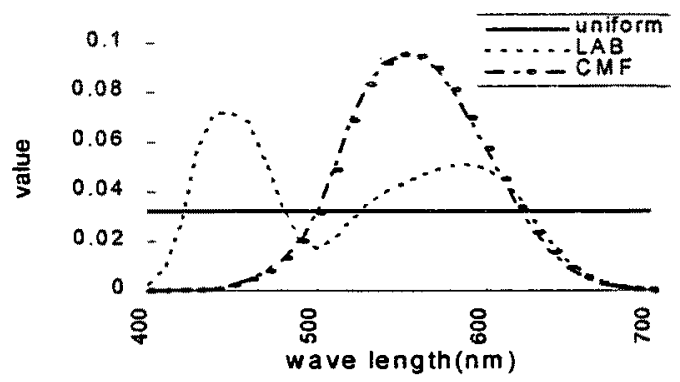

Fig. 1 Example of the HVWFs with uniform sensitivity, CMF, and LAB.

\subsection{Basis Functions Using HVWFs}

Reflectance set weighted with the HVWF produces weighted spectra set $S_{h}$ : $S_{h}=H_{\mathrm{v}} R$

where entries of diagonal matrix $\boldsymbol{H}_{\mathrm{v}}$ are values of HVWF and $\boldsymbol{R}$ is 31 by 1 . For deriving new basis vectors for the spectra weighted by HVWF $S_{h}$, SVD performed to minimize the error in the human visual domain:

$$
C_{h}=(1 / \mathrm{p}) \cdot S_{h} S_{h}^{+}=U_{h} D_{h} V_{h}^{+} \text {, }
$$

where $C_{h}$ is a correlation matrix of the spectra weighted by HVWF and $D_{h}$ is a diagonal matrix. The first $d$ columns of $U_{h}$ are orthonormal basis vectors for the linear model $\boldsymbol{B}_{h}$. The 690 reflectance set is used for obtaining these vectors. Figure 2 represent the first four basis vectors for $S R$ weighted by $L A B_{p=2}$. The feature of the $\mathrm{LAB}$ basis vector is different from the conventional and $\mathrm{CMF}$ cases [10]. The relation between a matrix of original SR $S$ and a reconstructed one $S^{\prime}$ becomes as:

$$
\boldsymbol{S}^{\prime}=\boldsymbol{B}_{h} \boldsymbol{W}_{h}=\boldsymbol{B}_{h}\left(\boldsymbol{B}_{h}{ }^{+} \boldsymbol{S}\right)=\left(\boldsymbol{B}_{h} \boldsymbol{B}_{h}{ }^{+}\right) \boldsymbol{S}=\boldsymbol{P}_{h} \boldsymbol{S}
$$

Let a matrix for mapping the original spectra $S$ onto the reconstructed spectra $S^{\prime}$ be the projection matrix $P_{h}$, via the basis vectors. The spectral reflectance estimated from the reconstructed spectra $S$ can be obtained by:

$$
\mathrm{R}^{\prime}(\lambda)=\sum_{j=1}^{d} B_{h j}(\lambda) W_{h j} / \operatorname{HVWF}(\lambda) .
$$

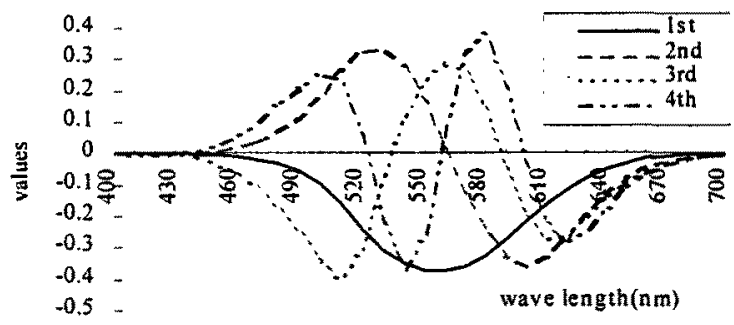

Fig. 2 The first four basis vectors for SR weighted by LAB

The mean error $E_{c}$ between the original and the approximated spectra is defined as:

$$
E_{h}=\left\|S-P_{h} \boldsymbol{S}\right\| \text {. }
$$


The HVWF in equation (24) is found at each term of $S^{\prime}$ and $S$ : diag. $\left(\boldsymbol{H}_{\nu}\right) \boldsymbol{R}^{\prime}=\boldsymbol{P}_{h}$ diag. $\left(\boldsymbol{H}_{\nu}\right) \boldsymbol{R}$.

Equation (27) can be rewritten by removing $H_{\nu}$ in each term: $\boldsymbol{R}^{\prime}=\boldsymbol{P}_{h} \boldsymbol{R}$.

\section{Experiments and Discussion}

A reflectance data set of 690 materials was selected for our experiments. The 690 SR data set consists of the following materials: 1) 400 color chips picked from Munsell color book measured using a PhotoResearch-703 spectro-radiometer; 2) 120 paint chips which selected from the Solid Selection of DuPont Color Sample, 170 natural and man-made objects, including rocks, plants and vegetation, human skin and hair, and fabrics of which complete data are available in ftp.eos.ncsu.edu [7]. In our experiment, we select the data set measured at $10 \mathrm{~nm}$ interval in $400-700 \mathrm{~nm}$ range. The averaged errors of reconstructed SR is estimated by the square root of equation (4) and the color differences on $X Y Z$ and LAB color spaces of the reconstructed SR can be estimated under the illuminant $E$, respectively [8].

Table 1 The errors for 3 7 Dimensional Reconstruction in the case of the CMF

\begin{tabular}{|c|c|c|c|c|c|c|c|}
\hline \multirow{2}{*}{$\begin{array}{c}\text { Basis } \\
\text { Dimen }\end{array}$} & \multicolumn{3}{|c|}{ ERROR } \\
\cline { 2 - 8 } -sion & \multicolumn{2}{|c|}{ LAB } & \multicolumn{2}{c|}{ XYZ } & \multicolumn{3}{c|}{ Ref. } \\
\cline { 2 - 8 } & avg. & max. & avg. & max. & avg. & max. & $1^{\text {st E.V.(\%) }}$ \\
\hline 3 & 2.931745 & 28.57438 & 0.914159 & 6.413741 & 0.027008 & 0.12995 & 99.11 \\
4 & 1.665761 & 14.59375 & 0.398795 & 3.818641 & 0.01715 & 0.106929 & 99.62 \\
5 & 1.070622 & 14.59240 & 0.272975 & 2.981871 & 0.01314 & 0.076783 & 99.84 \\
6 & 0.554749 & 6.638414 & 0.213997 & 2.654337 & 0.011001 & 0.074365 & 99.91 \\
7 & 0.491418 & 6.068511 & 0.138272 & 1.923089 & 0.008812 & 0.065608 & 99.96 \\
\hline
\end{tabular}

Table 1 summarizes the error distributions of three cases for the CMF. The errors are estimated under the five CIE standard illuminants, $D_{50}, D_{55}, D_{65}, D_{75}$, and $A$ for 690 $\mathrm{SR}$ using three-seven dimensional basis. The range of $\mathrm{XYZ}$ error distribution is under 0.92 and the LAB errors show below 2.93. Table 2 represents the details of the errors for 3-7-Dimensional reconstruction in LAB case. Table 1 and Table 2 show that the reconstuctitve ability of the LAB case is better than the CMF case.

Table 2. The errors for 3 7 Dimensional Reconstruction in the case of $\mathrm{LAB}_{\mathrm{g}=2}$

\begin{tabular}{|c|c|c|c|c|c|c|c|}
\hline \multirow{2}{*}{$\begin{array}{c}\text { Basis } \\
\text { Diemsion }\end{array}$} & \multicolumn{3}{|c|}{ ERROR } \\
\cline { 2 - 8 } & \multicolumn{2}{|c|}{ LAB } & \multicolumn{2}{c|}{ XYZ } & \multicolumn{3}{c|}{ Ref. } \\
\cline { 2 - 7 } & avg. & max. & avg. & max. & avg. & max. & lst E.V.(\%) \\
\hline 3 & 1.6989 & 15.0783 & 0.5555 & 3.3592 & 0.0258 & 0.1248 & 98.96 \\
4 & 1.2340 & 18.4376 & 0.4621 & 2.5695 & 0.0171 & 0.1034 & 99.60 \\
5 & 0.9171 & 13.7532 & 0.2967 & 2.9619 & 0.0123 & 0.0731 & 99.83 \\
6 & 0.5913 & 6.8157 & 0.2379 & 2.9637 & 0.0108 & 0.0731 & 99.90 \\
7 & 0.5075 & 5.4517 & 0.1716 & 1.9063 & 0.0086 & 0.0652 & 99.95 \\
\hline
\end{tabular}

Table 3 shows mean and maximum errors by three dimensional basis for three kinds of HVWFs. There is no serious difference among mean reflectance errors but maximum error has minimum value at uniformed $\mathrm{HVWF}$. In the case of the $\mathrm{LAB}_{\mathrm{p}=2}$, the average 
$L A B$ error of the reconstructed SR data under five different standard illuminants was 1.569018 that shows the best reproduction in our experiments. It is clear that the LAB error of uniformed HVWF is about 5.7, so the basis vectors of conventional type is difficult to be used for the purpose of special applications which need color discrimination between the original spectra and the reconstructed spectra. We can make a conclusion from table 1 , table2, and table 3 that the basis vectors derived from the LAB reconstruct the most accurate colors in our experiments.

Table 3 Errors estimated by the linear models using 3-D basis for 690 data set

\begin{tabular}{|c|c|c|c|c|c|c|}
\hline \multirow{2}{*}{ HVWFs } & \multicolumn{3}{|c|}{ ERROR } \\
\cline { 2 - 7 } & \multicolumn{2}{|c|}{ LAB } & \multicolumn{2}{c|}{ XYZ } & \multicolumn{2}{c|}{ Ref. } \\
\cline { 2 - 7 } & avg. & max. & avg. & max. & avg. & max. \\
\hline Uniform & 5.750791 & 52.085278 & 1.569018 & 11.654885 & 0.021617 & 0.101884 \\
CMF & 2.931745 & 28.574381 & 0.914159 & 6.413741 & 0.027008 & 0.12995 \\
LAB & 1.698997 & 15.078359 & 0.555544 & 3.359257 & 0.025865 & 0.1248 \\
\hline
\end{tabular}

ILLUMINATION EFFECT IN THE LINEAR MODEL:

The color differences of the LAB unit of the 690 spectrum reconstructed by each linear model for five standard illuminants are given in table 4 in the case of three dimensional basis vectors. The $C M F\left(D_{65}\right)$ is the $C M F$ estimated by equation (10) under the illuminant $D_{65}$ and $L A B\left(D_{65}\right)$ is the case of the $L A B$ estimated in $D_{65}$. CIE $D_{65}$ standard illuminant is assumed as a representative of illuminant. Table 4 summarizes the effect of the representative illuminant in linear models for the case of three dimensional basis. We can observe that the illumination effect on $C M F\left(D_{65}\right)$ is very serious while the representative of illumination of $L A B\left(D_{65}\right)$ display the worse effect than the uniformed illuminant of $L A B$. The main reason for the difference of illumination effect is, we think, that the illumination in equation (21) is non-linear to $\mathrm{LAB}$. The mean $\mathrm{LAB}$ color difference of $\mathrm{CMF}\left(\mathrm{D}_{6 s}\right)$ reduced to about $70 \%$ of the that of the CMF case except for the illuminant " $A$ " with about $111 \%$, which is due to the characteristic of representative illuminant. This gives us another important conclusion again that, basically, basis vectors in the linear model have to be derived independent of illumination characteristic. But carefully selected representative illuminant for the special purpose can increase the accuracy of representation of the linear model for SR data. For example, if we consider the linear model under the various day lights, it is easy to guess that the selection of proper representative illuminant will support to increase the accuracy. We can also know from the experiments for varying illuminant that the proposed linear model can be applied in reconstruction of color signals that are the product of spectral reflectance and illuminant with the same accuracy.

Table 4 Illuminant effect in linear models in the case of three dimensional basis

\begin{tabular}{|c|c|c|c|c|c|}
\hline \multirow{2}{*}{ Illuminant } & \multicolumn{5}{|c|}{ HVWFs } \\
\cline { 2 - 6 } & uniformed & CMF & CMF(D 65$)(\%$ of CMF) & LAB & LAB(D 65$):$ \\
\hline $\mathrm{D}_{50}$ & 5.633101 & 2.63924 & $1.8940971 .59 \%)$ & 1.36240 & 1.38171 \\
$\mathrm{D}_{55}$ & 6.00571 & 2.91406 & $2.09597(71.72 \%)$ & 1.42634 & 1.44550 \\
$\mathrm{D}_{65}$ & 6.35477 & 3.28648 & $2.42434(73.63 \%)$ & 1.57878 & 1.59586 \\
$\mathrm{D}_{75}$ & 6.61387 & 3.59474 & $2.71411(75.48 \%)$ & 1.75749 & 1.76890 \\
$\mathrm{~A}$ & 4.14648 & 2.22418 & $2.48798(111.5 \%)$ & 2.36995 & 2.59246 \\
\hline
\end{tabular}




\section{Conclusions}

In this paper, we have proposed color linear model, which explicitly takes into account human visual perception. Two kinds of HVC are derived from CIE color matching functions and LAB representation of the color matching functions. Those have been used for the reconstruction of spectral data sets of natural objects and the errors of reconstruction have been analyzed in terms of reflectance, XYZ, and LAB values. Through extensive experiments using 690 samples we observed that the proposed color linear models are superior to conventional linear model for the color representation of SR. The carefully selected representative illuminant increases the accuracy of the linear model for SR and the linear model can be applied in reconstruction of color signals with the high accuracy from the observation of the illumination effect.

\section{References}

1. J. Cohen, Dependency of the spectral reflectance curves of Munsell Color chips, Psychnomic Sci. 1, 367-370 (1964).

2. J. Parkkinen, J. Hallikainen, and T, Jaaskelainen, Characteristic spectra of Munsell colors, J. Opt. Soc. Am. A, 6, 318-322 (1989).

3. L. T. Maloney, Evaluation of linear models of surface spectral reflectance with small numbers of parameters, Color Res. \& Appl. 14, 325-334 (1986).

4. L. T. Maloney and B. A. Wandell, Color constancy; A method for recovering surface spectral reflectance, J. Opt. Soc. Am. A, 3, 29-33 (1986).

5. M. J. Vrhel and H. J. Trussell, Color Correction using principal components, Color Res. \& Appl., 17, 328-338 (1992).

6. D. Marimont and B. A. Wandell, Linear models of surface and illuminant spectra, J. Opt. Soc. Am. A, Vol9, No.11, Nov., 1905-1913 (1992).

7. M. J. Vrhel, R. Gershon, and L. S. Iwan, Measurement and Analysis of Object reflectance Spectra, Color Res. \& Appl. 19, 4-9 (1994).

8. G. Wyszecki and W. S. Stiles, Color Science 2nd Ed., John Wiley \& Sons (1982).

9. W.H. Press, S.A. Teukolsky, W.T. Vetterling and B.P. Flannery, Numerical Recipes in C-The Art of Scientific Computing, Cambridge Univ. Press (1992).

10. S. D. Lee, C. Y. Kim, and Y. S. Seo, Linear Model of Surface and Scanner Characterization Method, in IS\&T/SPIE's Symposium on Electronic Imaging : Device Independent Color Imaging II, Feb., San Jose, California, 84-93 (1995).

11. T. Jaakelainen, J. Parkkinen, and S. Toyooka, Vector-subspace Model for color representation, J. Opt. Soc. Am. A, Vol. 7, No. 4, April,725-730 (1990). 